\title{
THE GROWTH OF SUBUNIFORM ULTRAFILTERS
}

\section{S. NEGREPONTIS( $\left.{ }^{1}\right)$}

ABSTRACT. Some of the results on the topology of spaces of uniform ultrafilters are applied to the space $\Omega\left(a^{+}\right)$of subuniform ultrafilters (i.e., the set of ultrafilters which are $a$-uniform but not $a^{+}$-uniform) on $a^{+}$when $\alpha$ is a regular cardinal. The main object is to find for infinite cardinals $a$, such that $a=a^{a}$, a topological property that separates the space $\beta\left(\Omega\left(\alpha^{+}\right)\right) \backslash \Omega^{\prime} a^{+}$) (the growth of $\Omega\left(\alpha^{+}\right)$) from the space $U\left(\alpha^{+}\right)$of uniform ultrafilters on $\alpha^{+}$. Property $\Phi_{\alpha}$ fulfils this rôle defined for a zero-dimensional space $X$ by the following condition: every nonempty closed subset of $X$ of type at most $a$ is not contained in the uniform closure of a family of a pairwise disjoint nonempty open-and-closed subsets of $X$. The "infinitary" properties of $\Omega\left(a^{+}\right)$, as they are measured by $\Phi_{a}$, are more closely related to those of $U(a)$ than to those of $U\left(a^{+}\right)$. A consequence of this topological separation is that the growth of $\Omega\left(a^{+}\right)$is not homeomorphic to $U\left(a^{+}\right)$ and, in particular, that $\Omega\left(a^{+}\right)$is not $C^{*}$-embedded in the space $\Sigma\left(\alpha^{+}\right)$of $\alpha$-uniform ultrafilters on $a^{+}$. The se results are related to, and imply easily, the Aronszajn-Specker theorem: if $\alpha=a^{a}$ then $a^{+}$is not a ramifiable cardinal. It seems possible that similar questions on the $C^{*}$-embedding of certain spaces of ultrafilters depend on (and imply) results in partition calculus.

Property $\Phi_{a}$ was introduced in [7], where it was proved by a diagonal argument that the space $U(\alpha)$ of uniform altrafilters on $\alpha$ has property $\Phi_{\alpha}$ (Theorem 4.2 of [7]). It is easy to see that $U(\alpha)$ does not have property $\Phi_{\beta}$ for regular cardinals $\beta, \omega \leq \beta<\alpha$ (Lemma 3.3). The main result of the present paper (whose proof relies on Theorem 4.2 of [7]) is that if $\alpha=\alpha^{a}$ then the space $\beta\left(\Omega\left(\alpha^{+}\right)\right) \backslash \Omega\left(\alpha^{+}\right)$has property $\Phi_{\alpha}$.

Informally we think of this result as showing the inability of the space $\Omega\left(\alpha^{\dagger}\right)$ to break away from certain infinitary properties characteristic to $\alpha$ ( such as $\Phi_{\alpha}$ ), which are local after all, and to adopt the corresponding ones characteristic to $\alpha^{+}$(such as $\Phi_{\alpha^{+}}$), in the process of "going to infinity". Instead, under the constraint of a pigeon-hole principle (contained in Lemma 2.1 and in the proof of

Presented to the Society, May 24, 197 1; received by the editors May 17, 1971. AMS (MOS) subject classifications (1970). Primary 02K 35, 04-XX, 54G05.

Key words and phrases. Space of uniform, of subuniform ultrafilters, growth, property $\Phi_{a}, F_{\alpha}$-spaces, pigeon-hole principle, diagonal argument, normality, $C^{*}$-embedding, ramifiable cardinal, Aronszain-Specker theorem.

(1) The author wishes to acknowledge partial support received from the Canadian National Research Council under Grant A-4035 and from the 1972 Summer Research Institute of the Canadian Mathematical Congress. 
Theorem 3.2) the process of going to infinity is so "slow" that the local properties of $\Omega\left(\alpha^{+}\right)$(such as $\Phi_{\alpha}$ ), normally of little importance in determining the properties of the growth of a space, become the dominant factor.

An immediate consequence of the main theorem is that if $\alpha=\alpha^{\alpha}$ then $\Omega\left(\alpha^{+}\right)$ is not $C^{*}$-embedded in $\beta\left(\alpha^{+}\right)$(Corollary 3.4). For $\alpha=\omega$ this (together with Corollary 3.5, below, for $\alpha=\omega$ ) was a problem proposed in [1], [3], and proved by Nancy M. Warren in [9], [10] .

1. Notation and terminology. The axiom of choice is assumed. Ordinal numbers will be denoted by $\xi, \zeta, \eta, \sigma$. An ordinal is the set of all smaller ordinals, i.e. $\xi<\zeta$ is equivalent to $\xi \in \zeta$. Nevertheless, we will make the notational distinction between 0 (the first ordinal) and $\varnothing$ (the empty set). A cardinal number is an initial ordinal. Cardinals will be denoted by $\alpha, \beta, \gamma, 0,1, \ldots, n, \ldots$ denotes the sequence of natural numbers. The first infinite cardinal is $\omega$. The least cardinal greater than $\alpha$ is denoted by $\alpha^{+}$. $\alpha$ is a limit cardinal if it is not equal to $\beta^{+}$for some $\beta$. A cardinal is regular if it is not equal to the sum of fewer smaller cardinals. In this paper $\alpha$ will always denote an infinite regular cardinal. A nonlimit cardinal is regular. $\beta^{a}$ denotes the cardinal number of the set of all mappings from $\alpha$ to $\beta$. We set $\alpha^{\beta}=\Sigma\left\{\alpha^{\gamma}<\beta\right\}$. This cardinality of a set $A$ will be denoted by $|A|$. A set of cardinality $\alpha$ will usually be identified with $\alpha$ itself. For a set $A$, let $S(A), S_{\alpha}(A)$ be the set of all subsets of $A$, the set of all subsets of $A$ of cardinality less than $\alpha$, respectively. Note that for $\beta \leq \alpha,\left|S_{\beta}(\alpha)\right|=\alpha{ }^{\beta}$. The notion of an ultrafilter on $\alpha$ will be the usual one. An ultrafilter containing a set with a single element is called principal; otherwise nonprincipal. The set of all ultrafilters on $\alpha$, topologized with the Stone topology, and called the Stone-Cech compactification of $\alpha$, will be denoted by $\beta(\alpha)$. The set of principal ultrafilters in $\boldsymbol{\beta}(\alpha)$ is identified with $\alpha$ in the natural way. An ultrafilter on $\alpha$ is $\kappa$-uniform if each of its elements has cardinality at least $\kappa$. An $\alpha$-uniform ultrafilter on $\alpha$ is simply called uniform. The set of all uniform ultrafilters on $\alpha$, topologized as a subspace of $\beta(\alpha)$, is denoted by $U(\alpha)$. An ultrafilter on $\alpha^{+}$will be called subuniform if each of its elements has cardinality at least $\alpha$, and there is an element of the ultrafilter with cardinality $\alpha$. The set of all subuniform ultrafilters on $\alpha^{+}$, topologized as a subspace of $\beta\left(\alpha^{+}\right)$, is denoted by $\Omega\left(\alpha^{+}\right)$. We set $\Sigma(\alpha)=U\left(\alpha^{+}\right) \cup \Omega\left(\alpha^{+}\right)$, again a subspace of $\beta\left(\alpha^{+}\right)$.

Let $X$ be a (completely regular Hausdorff topological) space. For $Y \subset X$, we set $\mathrm{cl}_{X} Y$ for the closure of $Y$ in $X$. A zero-set $Z$ in $X$ is a set of the form $Z=\{x \in X: f(x)=0\}$ for some real-valued continuous function $f$ on $X$. The set of all bounded real-valued continuous functions on $X$ is denoted by $C^{*}(X)$. A subset $Y$ of $X$ is $C^{*}$-embedded in $X$ if for $f \in C^{*}(Y)$, there is $g \in C^{*}(X)$ such that $g \mid Y=f$. As with sets, $\beta X$ denotes the Stone-Čech compactification of $X$. A space 
$X$ is called totally disconnected if for any two distinct elements of $X$ there is an open-and-closed subset of $X$ containing one but not the other element; $X$ is called zero-dimensional if for any two disjoint zero-sets of $X$ there is an open-and-closed subset of $X$ containing one and disjoint from the other zero-set. Let $U$ be an open subset of a totally disconnected space $X$; the type $\tau(U)$ of $U$ is the least cardinal $\beta$ such that $U$ is equal to the union of $\beta$ open-and-closed subsets of $X$. If $F$ is a closed subset of $X$, we set $\tau(F)=r(X \backslash F)$. We note that if $F$ is a closed subset of a compact, zero-dimensional space, then $F$ is a zero-set if and only if $r(F) \leq \omega$. A totally disconnected space $X$ will be called an $F_{\alpha}$-space if every open subset of $X$ of type less than $\alpha$ is $C^{*}$-embedded in $X$. We remark that the usual notion of an $F$-space (meaning that every cozero set is $C^{*}$-embedded) is, in general, stronger than the notion of an $F_{\omega^{+}}$-space, but that the two notions coincide for compact spaces. (The reason for choosing this definition for an $F_{a}$-space, rather than the exact analogue of an $F$-space to higher cardinals, is only one of convenience.)

2. In this section we prove some results that will be needed for the proof of the main theorem (given in $\$ 3$ ).

2.1. Lemma. Let $F$ be a nonempty subset of $\beta\left(\Omega\left(a^{+}\right)\right)$, such that $F$ is equal to the intersection of at most a open-and-closed subsets of $\beta\left(\Omega\left(\alpha^{+}\right)\right)$. Then $F \cap \Omega\left(\alpha^{+}\right)$is nonempty.

Proof. Let $F=\bigcap_{\eta<\alpha^{2}} F_{\eta}$, where $F_{\eta}$ is open-and-closed in $\beta\left(\Omega\left(\alpha^{+}\right)\right)$. Set $U_{\eta}=\Omega\left(\alpha^{+}\right) \backslash F_{\eta}, \eta<\alpha$. If $F \cap \Omega\left(\alpha^{+}\right)=\varnothing$, then $\left\{U_{\eta}: \eta<\alpha\right\}$ is an open cover of $\Omega\left(a^{+}\right)$. Let $\Omega\left(a^{+}\right)$be equal to $\bigcup_{\xi<\alpha^{+}} S_{\xi}$, where

$S_{\xi} \subset S_{\zeta}$ for $\xi<\zeta<a^{+}$,

$S_{\xi}$ is open-and-closed in $\Omega\left(\alpha^{+}\right)$,

$S_{\xi}$ is homeomorphic to $U(\alpha)$

for $\xi<\alpha^{+}$. Let $\phi: \alpha^{+} \rightarrow S_{\omega}(\alpha)$ be any mapping such that for $\xi<\alpha^{+}$, $\bigcup\left\{U_{\eta}: \eta \in \phi(\xi)\right\} \supset S_{\xi}$. Then, there is $A \in S_{\omega}(\alpha)$, such that $\left|\left\{\xi<\alpha^{+}: \phi(\xi)=A\right\}\right|=$ $\alpha^{+}$. Clearly, then, $\bigcup\left\{U_{\eta}: \eta \in \dot{A}\right\}=\Omega\left(\alpha^{+}\right)$, contradicting the fact that $F$ is nonempty.

2.2. Corollary. Let $F_{\eta}$ be open-and-closed sets in $\Omega\left(a^{\dagger}\right), \eta<a$. Then

$$
\operatorname{cl}_{\beta\left(\Omega\left(a^{+}\right)\right)}\left(\bigcap_{\eta<a} F_{\eta}\right)=\bigcap_{\eta<a} \operatorname{cl}_{\beta\left(\Omega\left(a^{+}\right)\right)} F_{\eta} .
$$

Proof. We only need to prove that

$$
\operatorname{cl}_{\beta\left(\Omega\left(a^{+}\right)\right)}\left(\bigcap_{\eta<\alpha} F_{\eta}\right) \supset \bigcap_{\eta<\alpha} \operatorname{cl}_{\beta\left(\Omega\left(\alpha^{+}\right)\right)} F_{\eta} .
$$

Let $p \in \bigcap_{\eta<a} \operatorname{cl}_{\beta\left(\Omega\left(\alpha^{+}\right)\right)} F_{\eta} \backslash \operatorname{cl}_{\beta\left(\Omega\left(\alpha^{+}\right)\right)}\left(\bigcap_{\eta<\alpha} F_{\eta}\right)$. Then there is an open-andclosed set $N$ of $\boldsymbol{\beta}\left(\Omega\left(\alpha^{+}\right)\right)$, such that $p \in N$ and $N \cap \operatorname{cl}_{\beta\left(\Omega\left(\alpha^{+}\right)\right)}\left(\cap_{\eta<a} F_{\eta}\right)=\varnothing$. 
Then $F=N \cap \bigcap_{\eta<\alpha} \mathrm{cl}_{\beta\left(\Omega\left(\alpha^{+}\right)\right)} F_{\eta}$ is the intersection of at most $\alpha$ open-andclosed subsets of $\beta\left(\Omega\left(\alpha^{+}\right)\right)$, with $p \in F$; however, $F \cap \Omega\left(\alpha^{+}\right) \subset N \cap \bigcap_{\eta<a} F_{\eta}=\varnothing$, contradicting Lemma 2.1.

The following theorem has been proved as Theorem 3.1 in [5]. (We recall that in the present paper $\alpha$ is an infinite regular cardinal.)

2.3. Theorem. $U(\alpha)$ is a compact $F_{\alpha^{+}-\text {space. }}$

We will now prove a stronger result for the space $\Sigma\left(a^{+}\right)$of all uniform and subuniform ultrafilters on $\alpha^{+}$. The proof of the following lemma is left to the reader.

2.4. Lemma. Let $S=D \cup T$ be a space, where $D$ is discrete in $S$ and $T$ is compact. Then $S$ is a paracompact (and bence normal) space.

2.5. Theorem. $\Sigma\left(a^{+}\right)$is a compact $F a^{+-s p a c e . ~}$

Proof. It is clear that $\Sigma\left(a^{+}\right)$is a compact, zero-dimensional space. The Boolean algebra of open-and-closed subsets of $\Sigma\left(\alpha^{+}\right)$is (isomorphic to) $S\left(\alpha^{+}\right) / S_{\alpha}\left(a^{+}\right)$, an $\alpha$-complete Boolean algebra; thus, it is clear that the closure of any open subset of $\Sigma\left(\alpha^{+}\right)$of type less than $\alpha$ is open. It follows easily (e.g. as in Corollary 2.2 of [5]) that an open subset of $\Sigma\left(\alpha^{+}\right)$of type less than $\alpha$ is $C^{*}$-embedded in $\Sigma\left(a^{+}\right)$. Thus, we only have to prove that if $G$ is an open subset of $\Sigma\left(a^{+}\right)$of type (exactly) $\alpha$, then $G$ is $C^{*}$-embedded in $\Sigma\left(\alpha^{+}\right)$.

Let $G=\bigcup_{\xi<a} A_{\xi}^{\prime}$, where $A_{\xi} \subset \alpha^{+}$and $A_{\xi}^{\prime}=\mathrm{cl}_{\beta\left(\alpha^{+}\right)} A_{\xi} \cap \Sigma\left(\alpha^{+}\right)$(see Lemma 2.8 below in this connection). We express $G$ as the increasing union of open sets of type less than $\alpha$, of a chain of length $\alpha$, in the natural way: let $G_{\xi}=\bigcup_{\eta \leq \xi} A_{\eta}^{\prime}$ for $\xi<a$. Thus, $G_{\xi}$ is open, $\tau\left(G_{\xi}\right)<\alpha, G_{\xi} \subset G_{\eta}$ for $\xi<\eta<\alpha$, $G=\bigcup_{\xi<\alpha} G_{\xi}$. By the remarks above, $\bar{G}_{\xi}\left(=\mathrm{cl}_{\Sigma\left(a^{+}\right)} G_{\xi}\right)$ is open-and-closed in $\Sigma\left(\alpha^{+}\right)$, and $G_{\xi}$ is $C^{*}$-embedded in $\bar{G}_{\xi}$ for $\xi<\alpha$. Let $H=\bigcup_{\xi<\alpha} \bar{G}_{\xi}$; then $H$ is open in $\Sigma\left(\alpha^{+}\right), \tau(H) \leq \alpha$ and $G$ is dense in $H$.

We prove that $G$ is $C^{*}$-embedded in $H$. Indeed, let $f \in C^{*}(G)$. Set $f_{\xi}=f \mid G_{\xi}$ for $\xi<a$. Let $g_{\xi}$ be the unique continuous extension of $f_{\xi}$ to $\bar{G}_{\xi}$. Further, for $\xi<\eta<\alpha$, we have that $f_{\xi} \subset f_{\eta}$, and since the extensions are unique, that $g_{\xi} \subset$ $g_{\eta}$. Let $g=\bigcup_{\xi<a} g_{\xi}$. Since $\bar{G}_{\xi}$ is open for $\xi<a, g$ is continuous.

It is clear that $\bar{G} \xi=\left(\bigcup_{\eta \leq \xi} A_{\xi}\right)^{\prime}$ for $\xi<\alpha$. Set $B_{\xi}=\bigcup_{\eta \leq \xi} A_{\eta}, B=$ $\bigcup_{\xi<a} B_{\xi}=\bigcup_{\xi<a} A_{\xi}$. Thus $B_{\xi} \subset B_{\eta}$ for $\xi<\eta<a$. We now prove that $H$ is $C^{*}$-embedded in $B \cup H$. Indeed, let $f \in C^{*}(H)$. Set $f_{\xi}=f \mid \bar{G}_{\xi}$ for $\xi<a$. We proceed by transfinite recursion. By 2.4, $B_{0} \cup \bar{G}_{0}$ is a normal space, and $\bar{G}_{0}$ is a closed subset of $B_{0} \cup \bar{G}_{0}$. Let $g_{0} \in C^{*}\left(B_{0} \cup \bar{G}_{0}\right)$ be an extension of $f_{0}$, such that

$$
\sup \left\{\left|g_{0}(x)\right|: x \in B_{0} \cup \bar{G}_{0}\right\}=\sup \left\{\left|f_{0}(x)\right|: x \in \in^{\prime} \bar{G}_{0}\right\} \text {. }
$$


Let $\xi<\alpha$ and suppose that for every $\eta<\xi$ we have defined $g_{\eta}$ such that $g_{\eta} \epsilon$ $C^{*}\left(B_{\eta} \cup \bar{G}_{\eta}\right), f_{\eta} \subset g_{\eta}$,

$$
\sup \left\{\left|g_{\eta}(x)\right|: x \in B_{\eta} \cup \bar{G}_{\eta}\right\}=\sup \left\{\left|f_{\eta}(x)\right|: x \in \bar{G}_{\eta}\right\},
$$

$g_{\zeta} \subset g_{\eta}$ for $\zeta<\eta<\alpha$. We define

$$
g_{\xi}^{*}=\left(\left(\bigcup_{\eta<\xi} g_{\eta}\right) \cup f_{\xi}\right): \bigcup_{\eta<\xi}\left(B_{\eta} \cup \bar{G}_{\eta}\right) \cup \bar{G}_{\xi} \rightarrow \mathbf{R}
$$

(where $\mathbf{R}$ denotes the space of real numbers).

We verify that $g_{\xi}^{*}$ is well defined: our inductive assumption implies that $\bigcup_{\eta<\xi} g_{\eta}$ is well defined on $\bigcup_{\eta<\xi}\left(B_{\eta} \cup \bar{G}_{\eta}\right)$ and $f_{\xi}$ is defined on $\bar{G}_{\xi}$; further,

$$
\left(\bigcup_{\eta<\xi}\left(B_{\eta} \cup \bar{G}_{\eta}\right)\right) \cap \bar{G}_{\xi}=\bigcup_{\eta<\xi} \bar{G}_{\eta} \subset H
$$

and both functions $\bigcup_{\eta<\xi} g_{\eta}, f_{\xi}$ are equal to $f \mid\left(\bigcup_{\eta<\xi}\left(B_{\eta} \cup \bar{G}_{\eta}\right)\right) \cap G_{\xi}$ on the intersection. Thus $g_{\xi}^{*}$ is well defined. Further, $\bigcup_{\eta<\xi} g_{\eta}$ is continuous, since the sets $B_{\eta} \cup \bar{G}_{\eta}$ are open-and-closed for $\eta<\xi$. Finally, $g_{\xi}^{*}$ is continuous, because the sets

$$
\bigcup_{\eta<\xi} B_{\eta} \cup \overline{\bigcup_{\eta<\xi} G_{\eta}} \text { and } \bar{G}_{\xi} \backslash \overline{\bigcup_{\eta<\xi} G_{\eta}}
$$

are open-and-closed in $\bigcup_{\eta<\xi}\left(B_{\eta} \cup \bar{G}_{\eta}\right) \cup \bar{G}_{\xi}$. We note that the space $B \xi \cup \bar{G}_{\xi}$ is normal by Lemma 2.4 , and that $\cup_{\eta<\xi}\left(B_{\eta} \cup \bar{G}_{\eta}\right) \cup \bar{G}_{\xi}$ is a closed subset in $B_{\xi} \cup \bar{G}_{\xi}$. Further, notice that our inductive assumption implies that

$$
\sup \left\{\left|g_{\xi}^{*}(x)\right|: x \in \bigcup_{\eta<\xi}\left(B_{\eta} \cup \bar{G}_{\eta}\right) \cup \bar{G}_{\xi}\right\}=\sup \left\{\left|f_{\xi}(x)\right|: x \in \bar{G}_{\xi}\right\} .
$$

Hence $g_{\underline{\xi}}^{*}$ is bounded; thus, we can find a continuous extension $g_{\xi} \epsilon$ $C^{*}\left(B_{\xi} \cup \bar{G}_{\xi}\right)$, such that

$$
\sup \left\{\left|f_{\xi}(x)\right|: x \in \bar{G}_{\xi}\right\}=\sup \left\{\left|g_{\xi}(x)\right|: x \in B \xi \cup \bar{G}_{\xi}\right\} .
$$

This completes the recursive definition of $g_{\xi}$ for $\xi<\alpha$. We let $g=\bigcup_{\xi<a} g \xi$. Since the sets $B_{\xi} \cup \bar{G}_{\xi}$, $\xi<a$, are open-and-closed, $g$ is continuous. Also,

$$
\sup \{|g(x)|: x \in B \cup H\}=\sup \{|f(x)|: x \in B\},
$$

and hence $g$ is bounded. This completes the proof that $H$ is $C^{*}$-embedded in $B \cup H$.

Finally, we note that $B \subset B \cup H \subset \mathrm{cl}_{\beta(\alpha)}+B=\boldsymbol{\beta}(B)$. Hence, $B \cup H$ is $C^{*}$. embedded in $\mathrm{cl}_{\beta\left(a^{+}\right)}$. Since $\mathrm{cl}_{\beta\left(\alpha^{+}\right)} B$ is open-and-closed in $\beta\left(\alpha^{+}\right)$, it is $C^{*}$. embedded in $\beta\left(\alpha^{+}\right)$. Thus, by the transitivity of $C^{*}$-embedding, $G$ is $C^{*}$-embedded in $\beta\left(\alpha^{+}\right)$, and since it is contained in $\Sigma\left(\alpha^{+}\right)$, it is $C^{*}$-embedded in $\Sigma\left(\alpha^{+}\right)$. This completes the proof of the theorem.

The following result is a generalization of Theorem 4.1 by Fine and Gillman 
[3]; we remark that the reason for the additional restriction on the spaces $S_{\xi}$ below is not significant, and only reflects our restrictive (but convenient) definition of an $F_{a^{-s p a c e} \text {. }}$

2.6. Theorem. Let $X$ be an $F_{a^{-s p a c e}}$ and let $S \subset X$ be the union of a openand-closed subsets $S_{\xi}, \xi<\alpha$, of $X$, each $S_{\xi}$ being equal to the union of less than a compact subsets. Then

(a) $S$ is an $F_{a^{-s p a c e} \text {; }}$

(b) if $G \subset S$ and $G \cap S_{\xi}$ is of type less than a in $S$ for $\xi<\alpha$ then $G$ is $C^{*}$-embedded in $S$;

(c) if $X$ is zero-dimensional, then so is $S$.

Proof. (b) We may assume that $S=\bigcup_{\xi<a} S_{\xi}$ and that $S_{\xi} \subset S_{\zeta}$ for $\xi<\zeta<a$. We note, since each $S_{\xi}$ is an open subset of $X$ of type less than $\alpha$, that $S_{\xi}$ is an $F_{\alpha}$-space for $\xi<\alpha$. Let $g \in C^{*}(G)$, and set $g_{\xi}=g \mid G \cap S_{\xi}$ for $\xi<a$ Let $\zeta<\alpha$ and suppose that $g_{\xi}$ has been extended to $s_{\xi} \in C^{*}\left(s_{\xi}\right)$ for each $\xi<\zeta$, and that $s_{\zeta} \subset s_{\zeta^{\prime}}$ for $\zeta<\zeta^{\prime}<\xi$. The function $\bigcup_{\xi<\zeta^{s} \xi} \cup g_{\zeta}$ is well defined and continuous on the set $\bigcup_{\xi<\zeta} S_{\xi} \cup\left(G \cap S_{\zeta}\right)$, an open set of type less than $a$ in the $F_{a}$-space $S_{\zeta}$; hence it has an extension to a function $s_{\zeta} \in C^{*}\left(S_{\zeta}\right)$, such that

$$
\sup _{2}\left\{\left|s_{\zeta}(x)\right|: x \in S_{\zeta}\right\}=\sup \left\{\left|s_{\zeta}(x)\right|: x \in \bigcup_{\xi<\zeta} S_{\xi} \cup\left(G \cap S_{\zeta}\right)\right\} \text {. }
$$

Finally $\bigcup_{\xi<a} s_{\xi}$ is a continuous extension of $g$ to the space $S$.

(a) If $G$ is an open subset of $S$ of type less than $a$, then by (b), $G$ is $C^{*}$. embedded in $S$.

(c) We assume that $X$ is zero-dimensional. Let $A, B$ be two disjoint zerosets of $S$; we must prove that there is a $\{0,1\}$-valued continuous function on $S$, which is equal to 0 on $A$ and is equal to 1 on $B$. Let $A_{1}, B_{1}$ be open sets of $S$, with $r\left(A_{1} \cap S_{\xi}\right)<\alpha, \tau\left(B_{1} \cap S_{\xi}\right)<\alpha$ for $\xi<\alpha$, and such that $A \subset A_{1}, B \subset B_{1}$ $A_{1} \cap B_{1}=\varnothing$. (We can take $A_{1}, B_{1}$ to be disjoint cozero sets containing $A, B$, respectively; then $A_{1} \cap S_{\xi}, B_{1} \cap S_{\xi}$ are cozero sets in $S_{\xi}$; it is easy to see that the compactness condition on $S_{\xi}$ implies that $\tau\left(A_{1} \cap S_{\xi}^{*}\right)<\alpha, \tau\left(B_{1} \cap S_{\xi}\right)<\alpha$.) Let $g \in C^{*}\left(A_{1} \cup B_{1}\right)$ be equal to 0 on $A_{1}$ and to 1 on $B_{1}$. Note, since (by (a)) $S_{\xi}$ is $C^{*}$-embedded in $X$, that $S_{\xi}$ is zero-dimensional for $\xi<\alpha$. In the proof of (b), with $G=A_{1} \cup B_{1}$, we add to the recursive assumption that $\bigcup_{\xi<\zeta^{s} \xi}$ is $\{0,1\}$-valued; then $s_{\zeta}$ may be taken to be $\{0,1\}$-valued.

2.7. Corollary. $\Omega\left(\alpha^{+}\right)$is a zero-dimensional (locally compact) $F_{\alpha^{+-s p a c e . ~}}$

Proof. Apply 2.6(a), (c) with $X=\Sigma\left(\alpha^{+}\right)$, an $F_{\alpha^{+-s p a c e}}$ by $2.5, S=\Omega\left(a^{+}\right)$, and with $\alpha^{+}$replacing $\alpha$.

The following fact is also needed. 
2.8. Lemma. Let $X$ be a locally compact, zero-dimensional space, and let $F$ be an open-and-closed subset of $\beta X \backslash X$. Then there is an open-and-closed set $N$ in $X$, such that $N^{\prime}=\mathrm{cl}_{\beta X} N \backslash X=F$.

Proof. Since $X$ is zero-dimensional, $\beta X$ is also zero-dimensional. The family $\{V \cap(\beta X \backslash X): V$ open-and-closed in $\beta X\}$ forms a base for the topology of $\beta X \backslash X$. By the local compactness of $X, \beta X \backslash X$ is compact, and thus $F$ is equal to the union of a finite number of elements of the base. Thus $F=\left(V_{1} \cup \ldots \cup V_{n}\right) \cap$ $(\beta X \backslash X)$ where $n<\omega$ and $V_{i}$ is open-and-closed in $\beta X$ for $i \leq n$. We set $N=$ $\left(V_{1} \cup \cdots \cup V_{n}\right) \cap X$; it is clear that $N^{\prime}=F$.

2.9. Theorem. Let $F$ be a closed subset of $\beta\left(\Omega\left(\alpha^{+}\right)\right) \backslash \Omega\left(\alpha^{+}\right)$of type at most $\alpha$. Then there is a closed subset $W$ of $\Omega\left(\alpha^{+}\right)$, of type at most $\alpha$, and such that $W^{\prime}=\operatorname{cl}_{\beta\left(\boldsymbol{\Omega}\left(\alpha^{+}\right)\right)} W \backslash \Omega\left(\alpha^{+}\right)=F$.

Proof. Let $F=\bigcap_{\eta<\alpha} F_{\eta}$, where $F_{\eta}$ is open-and-closed in $\beta\left(\Omega\left(\alpha^{+}\right)\right) \backslash \Omega\left(\alpha^{+}\right)$ for $\eta<\alpha$. Since, by $2.7, \Omega\left(\alpha^{+}\right)$is locally compact and zero-dimensional, there is, by 2.8, an open-and-closed set $N_{\eta}$ in $\Omega\left(\alpha^{+}\right)$, such that $N_{\eta}^{\prime}=F_{\eta}$ for all $\eta<\alpha$. We set $W=\bigcap_{\eta<a} N_{\eta}$; thus $W$ is a closed subset of $\Omega\left(\alpha^{+}\right)$of type at most $\alpha$. By 2.2,

$$
\begin{aligned}
W^{\prime} & =\operatorname{cl}_{\beta\left(\Omega\left(a^{+}\right)\right)} W \backslash \Omega\left(a^{+}\right)=\operatorname{cl}_{\beta\left(\Omega\left(a^{+}\right)\right)}\left(\bigcap_{\eta<a} N_{\eta}\right) \backslash \Omega\left(a^{+}\right) \\
& =\bigcap_{\eta<\alpha}\left(\operatorname{cl}_{\beta\left(\Omega\left(a^{+}\right)\right)} N_{\eta}\right) \backslash \Omega\left(\alpha^{+}\right)=\bigcap_{\eta<\alpha} N_{\eta}^{\prime} \\
& =\bigcap_{\eta<\alpha} F_{\eta}=F .
\end{aligned}
$$

3. The main results. The following result, mentioned in the introduction, will be used.

3.1. Theorem. $U(\alpha)$ bas property $\Phi_{a}$. In detail, this means the following: Let $F$ be a closed, nonempty subset of $U(\alpha)$ of type at most $\alpha$, and let $\left\{V_{\eta}, \eta<\alpha\right\}$ be a family of pairwise disjoint, nonempty subsets of $U(\alpha)$; then, there is $p \in F$ and an open-and-closed set $N$ in $U(\alpha)$ such that $p \in N$ and $\left|\left\{\eta<\alpha: V_{\beta} \cap N \neq \varnothing\right\}\right|<\alpha$ (this last condition is expressed by saying that $p$ is not in the uniform closure of $\left.\left\{V_{\eta}, \eta<\alpha\right\}\right)$.

The proof of this theorem is given in Theorem 4.2(i) (together with 4.1(ii)) of [7].

We now state and prove the main theorem of this paper.

3.2 Theorem. If $\alpha=\alpha^{a}$ then $\beta\left(\Omega\left(\alpha^{+}\right)\right) \backslash \Omega\left(\alpha^{+}\right)$bas property $\Phi_{\alpha}$.

Proof. Let $F$ be a closed, nonempty subset of $\beta\left(\Omega\left(a^{+}\right)\right) \backslash \Omega\left(a^{+}\right)$, such that $F=\bigcap_{\eta<\alpha} F_{\eta}$, where $F_{\eta}$ is open-and-closed in $\beta\left(\Omega\left(\alpha^{+}\right)\right) \backslash \Omega\left(\alpha^{+}\right)$for $\eta<\alpha$. 
Let $\left\{V_{\eta}, \eta<a\right\}$ be a family of pairwise disjoint, nonempty open-and-closed subsets of $\beta\left(\Omega\left(\alpha^{+}\right)\right) \backslash \Omega\left(\alpha^{+}\right)$. By 2.7 and 2.8 , there are open-and-closed sets $N_{\eta}$ in $\Omega\left(\alpha^{+}\right)$such that $N_{\eta}^{\prime}=\operatorname{cl}_{\beta\left(\Omega\left(\alpha^{+}\right)\right)} N_{\eta} \backslash \Omega\left(\alpha^{+}\right)=V_{\eta}$ for $\eta<\alpha$. Let $\Omega\left(\alpha^{+}\right)$be equal to $\bigcup_{\xi<\alpha^{+}} S_{\xi}$, where

$S_{\xi} \subset S_{\zeta}$ for $\xi<\zeta<\alpha^{+}$,

$S_{\xi}$ is open-and-closed in $\Omega\left(\alpha^{+}\right)$for $\xi<\alpha^{+}$,

$s_{\xi}$ is homeomorphic to $U(\alpha)$ for $\xi<\alpha^{+}$.

Note that a subset of $\Omega\left(\alpha^{+}\right)$is relatively compact (i.e. it has compact closure) in $\Omega\left(\alpha^{+}\right)$if and only if it is a subset of $S_{\xi}$ for some $\xi<\alpha^{+}$. Thus $N_{\eta} \cap N_{\eta^{\prime}} \subset S_{\xi\left(\eta, \eta^{\prime}\right)}$ for some $\xi\left(\eta, \eta^{\prime}\right)<\alpha^{+}$, if $\eta<\eta^{\prime}<\alpha$. By taking $\xi$, such that $\xi\left(\eta, \eta^{\prime}\right)<\xi<\alpha^{+}$for all $\eta<\eta^{\prime}<a$, and by replacing $N_{\eta}$ by $N_{\eta} \backslash s_{\xi}$, we may assume that the family $\left\{N_{\eta}, \eta<a\right\}$ consists of pairwise disjoint sets. Further, we note that we may assume that $V_{\eta} \cap F=\varnothing$ for all $\eta<\alpha$; otherwise, any $p \in V_{\eta} \cap F$ (for some $\eta<\alpha$ ) and $N=V_{\eta}$ will satisfy the conclusion of the theorem.

By Theorem 2.9, there is a closed subset $W$ of $\Omega\left(a^{+}\right)$, of type at most $\alpha$, such that $W^{\prime}=\operatorname{cl}_{\beta\left(\Omega\left(a^{+}\right)\right)} W \backslash \Omega\left(\alpha^{+}\right)=F$. Then $\left(W \cap N_{\eta}\right)^{\prime}=W^{\prime} \cap N_{\eta}^{\prime}=F \cap V_{\eta}=\varnothing$ (using Corollary 2.2). Thus $W \cap N_{\eta} \subset S_{\xi(\eta)}$ for some $\xi(\eta)<\alpha^{+}$; in the same way, as above, we may assume that $W \cap N_{\eta}=\varnothing$ for $\eta<\alpha$. We set

$B=\left\{x \in W: x\right.$ is not in the uniform closure of the family $\left.\left\{N_{\eta}, \eta<a\right\}\right\}$.

Thus $x \in B$ if and only if there is some open-and-closed set $N(x)$ containing $x$, such that $\left|\left\{\eta<a: N(x) \cap N_{\eta} \neq \varnothing\right\}\right|<\alpha$. By Theorem 3.1, we conclude that $B$ is not relatively compact in $\Omega\left(\alpha^{+}\right)$. Indeed, if $B$ is relatively compact, then $B \subset S_{\xi}$ for some $\xi<\alpha^{+}$. Since $W$ is not relatively compact (because $W^{\prime}=F \neq \varnothing$ ), there is $\zeta, \xi<\zeta<\alpha^{+}$, such that $(W \backslash B) \cap\left(s_{\zeta} \backslash S_{\xi}\right) \neq \varnothing$. Thus $S_{\zeta} \backslash S_{\xi}$ is homeomorphic to $U(\alpha)$, and $W \backslash B$ is a nonempty closed subset of it, of type not exceeding $a$, every element of which is in the uniform closure of the family $\left\{N_{\eta} \cap\left(S_{\zeta} \backslash S_{\xi}\right): \eta<\alpha\right\}$, in contradiction to 3.1. By transfinite recursion, we can find a set $C=\left\{c_{\xi}, \xi<\alpha^{+}\right\}$and open-and-compact sets $N\left(c_{\xi}\right)$ in $\Omega\left(a^{+}\right)$for $\xi<\alpha^{+}$, such that $C \subset B,|C|=a^{+}$, if $D \subset C$, then $D$ is relatively compact if and only if $|D| \leq a, c_{\xi} \in N\left(c_{\xi}\right)$ for $\xi<\alpha^{+}, N\left(c_{\xi}\right) \cap N\left(c_{\zeta}\right)=\varnothing$ for $\xi<\zeta<\alpha^{+}$, and $\left|\left\{\eta<\alpha: N\left(c_{\xi}\right) \cap N_{\eta} \not \varnothing\right\}\right|<\alpha$ for $\xi<\alpha^{+}$. We set $A_{\xi}=\left\{\eta<\alpha: N\left(c_{\xi}\right) \cap N_{\eta} \neq \varnothing\right\}$, and let $\phi: \alpha^{+} \rightarrow S_{\alpha}(\alpha)$ be defined by $\phi(\xi)=A_{\xi}$. Since $\left|S_{\alpha}(\alpha)\right|=\alpha^{a}=\alpha$, there is $A \in S_{\alpha}(\alpha)$, such that $A=A_{\xi}$ for $\alpha^{+}$many $\xi<\alpha^{+}$. We set $D_{A}=\left\{\xi<\alpha^{+}: A=A_{\xi}\right\}$. We consider the set

$$
G=\bigcup_{\eta<\alpha}\left\{N_{\eta}: \eta \in \alpha \backslash A\right\} \cup \bigcup\left\{N\left(c_{\xi}\right): \xi \in D_{A}\right\} .
$$

We apply Theorem 2.6, with $X=S=\Omega\left(a^{+}\right)$, and with $G$ defined as above (with $\alpha^{+}$replacing $\left.\alpha\right)$. The condition $r\left(G \cap S_{\xi}\right) \leq \alpha$ is satisfied for $\xi<\alpha^{+}$. Thus $G$ is $C^{*}$-embedded in $\Omega\left(\alpha^{+}\right)$. Let $f \in C^{*}\left(\Omega\left(\alpha^{+}\right)\right)$be such that 


$$
\begin{aligned}
f(x)=1 & \text { if } x \in \bigcup_{\eta<a}\left\{N_{\eta}: \eta \in \alpha \backslash A\right\}, \\
=0 & \text { if } x \in \bigcup\left\{N\left(c_{\xi}\right): \xi \in D_{A}\right\} .
\end{aligned}
$$

Let $\bar{f} \in C\left(\beta\left(\Omega\left(\alpha^{+}\right)\right)\right.$be the continuous extension of $f$ to $\beta\left(\Omega\left(\alpha^{+}\right)\right)$. Let $p$ be any element of $\beta\left(\Omega\left(\alpha^{+}\right)\right) \backslash \Omega\left(\alpha^{+}\right)$in the closure of $\left\{c_{\xi}: \xi \in D_{A}\right\}$. Then, since $C \subset$ $B \subset W$ and $W^{\prime}=F$, we have $p \in F$; further, $\bar{f}(p)=0$. Also, if $q \in N_{\eta}^{\prime}=V_{n}$ for some $\eta \in \alpha \backslash A$, we have that $7(q)=1$. We set $N=\left\{p \in \beta\left(\Omega\left(\alpha^{+}\right)\right) \backslash \Omega\left(\alpha^{+}\right): \bar{f}(p) \neq 1\right\}$. Then $p \in N$ and $\left\{\eta<\alpha: V_{\eta} \cap N \neq \varnothing\right\} \subset A$, hence $\left|\left\{\eta<\alpha: V_{\eta} \cap N \neq \varnothing\right\}\right|<\alpha$. This completes the proof of the theorem.

For $\alpha=\omega$, Theorem 3.2 is easily seen to be equivalent to the statement: Every nonempty zero-set of $\beta\left(\Omega\left(\omega^{+}\right)\right) \backslash \Omega\left(\omega^{+}\right)$has nonempty interior. The reader will be able to give a simple direct proof of this statement, along the lines of the proof of Theorem 3.2, using only the following known facts: every nonempty zeroset of $\beta \omega \backslash \omega$ has nonempty interior; $\Omega\left(\omega^{+}\right)$is a pseudocompact, locally compact, zero-dimensional $F_{\omega^{+}}$-space; and Theorem 4.1 of [3]. Note that it follows that $\beta\left(\Omega\left(\omega^{+}\right)\right) \backslash \Omega\left(\omega^{+}\right)$is not basically disconnected.

3.3. Lemma. If $\alpha, \beta$ are regular cardinals and $\omega \leq \beta<\alpha$, then $U(\alpha)$ does not have property $\Phi_{\beta}$.

Proof. Let $d=\left(D_{\eta}\right)_{\eta<\beta}$ be a family of subsets of $\alpha$, such that $\left|D_{\eta}\right|=\alpha$ for $\eta<\beta, D_{\eta} \cup D_{\eta^{\prime}}=\varnothing$ for $\eta<\eta^{\prime}<\beta$, and $\cup_{\eta<\beta} D_{\eta}=\alpha$. Let $F=$ $\bigcap_{\zeta<\beta}\left(\alpha \backslash \bigcap_{\eta \leq \zeta} D_{\eta}\right)^{\prime}$ and $V_{\eta}=D_{\eta}^{\prime}$ for $\eta<\beta$. (We have set for $A \subset \alpha, A^{\prime}=$ $\left(\operatorname{cl}_{\beta(\alpha)} A\right) \cap U(\alpha)$.). Then $F$ is a closed, nonempty subset of $U(\alpha)$ of type at most $\beta$, and $\left\{V_{\eta}, \eta<\beta\right\}$ is a family of $\beta$ pairwise disjoint nonempty open-andclosed subsets of $U(\alpha)$. But it is clear that if $p \in F$ and $N$ is any open set of $U(\alpha)$ containing $p$, then $\left|\left\{\eta<\alpha: V_{\eta} \cap N \neq \varnothing\right\}\right|=\beta$.

3.4. Corollary. If $\alpha=\alpha^{a}$, then $\beta\left(\Omega\left(\alpha^{+}\right)\right) \backslash \Omega\left(\alpha^{+}\right)$is not bomeomorpbic to $U\left(\alpha^{+}\right)$. In particular $\Omega\left(\alpha^{+}\right)$is not $C^{*}$-embedded in $\beta\left(\alpha^{+}\right)$.

Proof. By Theorem 3.2, $\beta\left(\Omega\left(\alpha^{+}\right)\right) \backslash \Omega\left(\alpha^{+}\right)$has property $\Phi_{\alpha^{*}}$. By Lemma 3.3, (with $\alpha^{+}$, a replacing $\alpha, \beta$, respectively) $U\left(\alpha^{+}\right)$does not have property $\Phi_{\alpha^{*}}$

The following argument is the natural extension of the proof given for Corollary 2 in [9].

3.5. Corollary. Let $a=a^{a}$.

(a) If $\alpha^{+} \cup \Omega\left(\alpha^{+}\right) \subset X \subset \beta\left(a^{+}\right) \backslash U\left(\alpha^{+}\right)$, then $X$ is not a normal space; and

(b) $\Omega\left(\alpha^{+}\right)$is not a normal space.

Proof. (a) follows from the fact that $\Omega\left(\alpha^{+}\right)$is a closed subset of $X$ which is not $C^{*}$-embedded in $X$ (by 3.4). 
(b) It follows from (a) that $a^{+} \cup \Omega\left(a^{+}\right)$is not a normal space. To prove (b) it is sufficient to prove that there is a closed subset of $\Omega\left(\alpha^{+}\right)$which is homeomorphic to $\alpha^{+} \cup \Omega\left(\alpha^{+}\right)$. Let $\phi: \alpha^{+} \rightarrow \alpha^{+}$be the unique one-to-one, order-preserving function such that

$$
\phi\left[\alpha^{+}\right]=\left\{\xi<\alpha^{+}: c f(\xi)=\alpha\right\}
$$

We define

and we note that

$$
S_{\xi}=\left(\mathrm{cl}_{\beta\left(a^{+}\right)} \phi(\xi)\right) \cap \Omega\left(\alpha^{+}\right) \text {for } \xi<\alpha^{+}
$$

$\Omega\left(\alpha^{+}\right)=\bigcup_{\xi<\alpha^{+}} S_{\xi}$

$S_{\xi}$ is homeomorphic to $U(\alpha)$ for $\xi<\alpha^{+}$,

$S_{\xi}^{\zeta}$ is open-and-closed in $\Omega\left(a^{+}\right)$for $\xi<a^{+}$, and

$s_{\zeta} \varsubsetneqq S_{\xi}$ for $\zeta<\xi<\alpha^{+}$.

Let $\left\{H_{\xi}: \xi<\alpha^{+}\right\}$be a family of nonempty open-and-closed subsets of $\Omega\left(\alpha^{+}\right)$ such that $H_{\xi} \subset S_{\xi+1} \backslash S_{\xi}$ for $\xi<\alpha^{+}$and define $H=\bigcup_{\xi<a^{+}} H_{\xi}$. It follows from Theorem 2.6 and Corollary 2.7 that $H$ is an open subset of $\Omega\left(\alpha^{+}\right)$which is $C^{*}$. embedded in $\Omega\left(\alpha^{+}\right)$. Let $a_{\xi} \in H_{\xi}$ for $\xi<\alpha^{+}$and let $A=\left\{a_{\xi}: \xi<\alpha^{+}\right\}$. Then $A$ is a $C^{*}$-embedded subset of $\Omega\left(\alpha^{+}\right)$homeomorphic to $\alpha^{+}$. It is clear that if $B \subset A$ and $|B| \leq \alpha$ then $\mathrm{cl}_{\Omega\left(\alpha^{+}\right)} B$ is compact and hence homeomorphic to $\alpha^{+} \cup \Omega\left(\alpha^{+}\right)$. This completes the proof.

We remark that the argument given in part (b) of the above corollary proves that for any infinite regular cardinal $\alpha$ there is a closed subset of $\Omega\left(\alpha^{+}\right)$homeomorphic to $\alpha^{+} \cup \Omega\left(\alpha^{+}\right)$.

For the statement of the classical Aronszajn-Specker theorem, which follows from our results, we need the following definitions. A ramification system is a partially ordered set $\langle A, \preceq\rangle$ with a least element (if it is nonempty) and such that the set

$$
P(a)=\{x \in A: x \preceq a \text { and } x \neq a\}
$$

is well ordered by $\preceq$ for $a \in A$. The order of an element $a \in A_{0}$ is the order type of $P(a)$, i.e., the unique ordinal isomorphic to the well-ordered set $P(a)$. The order of the ramification system $\langle A, \preceq\rangle$ is the least ordinal $\xi$, such that the order of $P(a)<\xi$ for all $a \in A$. A cardinal $\alpha$ is ramifiable if every ramification system $\langle A, \preceq\rangle$ of order $\alpha$, such that

$\mid\{a \in A$ : order type of $P(a)=\xi\} \mid<\alpha$ for $\xi<\alpha$,

has a subset of cardinality $\alpha$ well ordered by $\preceq$.

3.6. Corollary (Aronszajn [4]; Specker [8]; Monk [2, p. 76]). If $a=a^{a}$ then $a^{+}$is not a ramifiable cardinal.

Proof. Since $\Omega\left(\alpha^{+}\right)$is zero-dimensional (Corollary 2.7), and $\Omega\left(\alpha^{+}\right)$is not $C^{*}$. embedded in $\beta\left(\alpha^{+}\right)$it follows that there is a $\{0,1\}$-valued continuous function $f$ 
on $\Omega\left(\alpha^{+}\right)$which cannot be extended to a $\{0,1\}$-valued continuous function on $\Sigma\left(\alpha^{+}\right)$. For every $\xi<\alpha^{+}$, we choose a $\{0,1\}$-valued function $f_{\xi}: \xi \rightarrow\{0,1\}$, such that

$$
\bar{f}_{\xi}\left|\Omega\left(\alpha^{+}\right) \cap \mathrm{cl}_{\beta\left(\alpha^{+}\right)} \bar{\xi}=f\right| \Omega\left(\alpha^{+}\right) \cap \mathrm{cl}_{\beta\left(\alpha^{+}\right)} \xi .
$$

Since $\Omega\left(\alpha^{+}\right) \cap \mathrm{cl}_{\beta\left(\alpha^{+}\right)} \xi$ is a compact subset of $\alpha^{+} \cup\left(\Omega\left(\alpha^{+}\right) \cap \mathrm{cl}_{\beta\left(\alpha^{+}\right)} \xi\right)$, such a a function $f_{\xi}$ exists for $\xi<\alpha^{+}$. For $\xi<\alpha^{+}$we set

$$
\mathfrak{A}_{\xi}=\left\{b \in\{0,1\}^{\xi}: b=f_{\eta} \mid \xi \text { for some } \eta \text { with } \xi \leq \eta<\alpha^{+}\right\} \text {, }
$$

and we set $\mathbb{A}=\bigcup_{\xi<a^{+}} \mathfrak{Q}_{\xi}$. We make $\mathfrak{A}$ into a partially ordered set by setting $b \leq b^{\prime}$ if $b \subset b^{\prime}$ (for $b$ and $b^{\prime}$ in $\mathfrak{Q}$ ). It is easy to verify that $\mathbb{Q}$ is a ramification system, that for $\xi<\alpha^{+}$the set of elements of $\mathbb{Q}$ of order $\xi$ is the set $\mathbb{A}_{\xi}$, and (since $f_{\xi} \in \mathbb{A}_{\xi}$ ) that $\mathbb{A}_{\xi} \neq \varnothing$ for $\xi<\alpha^{+}$. Furthermore, if $b \in \mathbb{Q}_{\xi}$, then $\left|\left\{\zeta<\xi: b(\zeta) \neq f_{\xi}(\zeta)\right\}\right|<\alpha$ (because $\bar{b}\left|\Omega\left(\alpha^{+}\right) \cap \mathrm{cl}_{\beta\left(\alpha^{+}\right)} \xi=\bar{f}_{\xi}\right| \Omega\left(\alpha^{+}\right) \cap \mathrm{cl}_{\beta\left(a^{+}\right)} \xi$; it then follows that $\left|\mathfrak{Q}_{\xi}\right| \leq \alpha^{a}=\alpha<\alpha^{+}$for $\xi<\alpha^{+}$). And finally, ( $\mathcal{H}^{+}$does not contain any well-ordered subset of order-type $\alpha^{+}$; indeed if $\left\{b_{\xi}: \xi<\alpha^{+}\right\}$were such a chain, then $b=\bigcup_{\xi<a^{+} b_{\xi}}$ would be a well-defined, $\{0,1\}$-valued function on $a^{+}$such that $f \subset \bar{b} \mid \Sigma\left(\alpha^{+}\right)$, contrary to the choice of $f$. This completes the proof of the theorem.

We finally remark that results similar to those leading to Theorem 3.2 hold for the growth of the space $S_{\alpha} \backslash\{p\}$, when $\alpha=\alpha^{a}, S_{a}$ is the Stone space of the $\alpha$ homogeneous, $\alpha$-universal Boolean algebra of cardinality $\alpha$, and $p$ is a $P_{a}$-point of $S_{a}$ (see [6] for the theory of these spaces). The details, which are similar and somewhat simpler to those of the present paper, will be given elsewhere.

\section{BIBLIOGRAPHY}

1. W.W. Comfort and S. Negrepontis, Homeomorphs of three subspaces of $\beta N \backslash N$, Math. Z. 107 (1968), 53-58. MR 38 \#2739.

2. P. Erdös and A. Tarski, On some problems involving inaccessible cardinals, Essays on the Foundation of Mathematics, Magnes Press, Jerusalem, 1967, pp. 50-82.

3. N. J. Fine and L. Gillman, Extension of continuous functions in $\beta N$, Bull. Amer. Math. Soc. 66 (1960), 376-381. MR 23 \#A619.

4. G. Kurepa, Ensembles linéaires et une classe de tableaux ramifies (tableaux ramifies de M. Aronszajn), Publ. Math. Univ. Belgrade 6 (1936), 129-160.

5. S. Negrepontis, Extension of continuous functions in $\beta D$, Nederl. Akad. Wetensch. Proc. Ser. A 71 = Indag. Math. 30 (1968), 393-400. MR 39 \#2128.

6. - The Stone space of the saturated Boolean algebras, Trans. Amer. Math. Soc. 141 (1969), 515-527. MR $40 \# 1311$.

7. - The existence of certain uniform ultrafilters, Ann. of Math. (2) 90 (1969), 23-32. MR 40 \#46.

8. E. Specker, Sur un problème de Sikorski, Colloq. Math. 2 (1949), 9-12. MR 12, 597.

9. N. M. Warren, Extending continuous functions in Stone.Čech compactifications of discrete spaces and in zero-dimensional spaces, Doctoral Dissertation, University of Wisconsin, Madison, Wis., 1970. 10. - Properties of the Stone-Čch compactifications of discrete spaces, Proc. Amer. Math. Soc. 33 (1972), 599-606.

DEPARTMENT OF MATHEMATICS, MCGILL UNIVERSITY, MONTREAL, QUEBEC, CANADA DEPARTMENT OF MATHEMATICS, ATHENS UNIVERSITY, ATHENS, GREECE (Current address) 